\title{
Optimal IIR Filter Based Equalizer Design via a Functional Inequality Constrained Optimization Approach
}

\author{
Yan Liang ${ }^{1}$, Ling Wing-Kuen ${ }^{1+}$ and $\mathrm{Ya} \mathrm{Li}^{2}$ \\ ${ }^{1}$ School of Information Engineering, Guangdong University of Technology, China \\ ${ }^{2}$ School of Information Engineering, Guangdong Polytechnic Normal University, China
}

\begin{abstract}
A finite impulse response (FIR) filter based equalizer requires a very high computational power and suffers from a long delay. On the other hand, an infinite impulse response (IIR) filter based equalizer can reduce a lot of computational cost for the implementation. This paper develops a joint model of a speaker and a transmission line via both the input audio signal and the input signal of the equalizer. The frequency response of the transmission system is modeled by the discrete time Fourier Transform of the input and output signal of the system. The modeled frequency response of the transmission system multiplies the frequency response of the equalizer should be approximately equal to 1 at every frequency point. To design these IIR filters, the total absolute errors between 1 and the modeled frequency response of the transmission system multiplies the frequency response of the corresponding IIR filters are minimized subject to the specifications on their maximum upper bounds and the stability condition of the equalizer. These problems are actually the functional inequality constrained optimization problems. By employing the constraint transcription method, these infinite constrained optimization problems can be converted to the finite constrained optimization problems and the solution is the coefficients of the IIR filter. The computer numerical simulation results show that the product of the frequency response of the developed joint model and that of the equalizer is small.
\end{abstract}

Keywords: equalizer design, infinite impulse response filter design, functional inequality constrained optimization, infinite constrained optimization, constraint transcription.

\section{Introduction}

An equalizer is usually used to adjust the gains of different frequency components of an audio system in order to attenuate the unwanted components of the signal such as the transmission noise from the background due to the sound field defects [1] and the distortion generated by the speaker [2]. As using the equalizer can significantly enhanced the performance of the audio system, the equalizer is widely used in our daily life such as in the music production and in a live broadcasting.

The equalizer is characterized by three parameters. They are center frequency, gain and bandwidth [3]. A parametric equalizer is the one in which divides the whole frequency range into several frequency segments and adjusts the center frequency, gain and bandwidth according to the instructions in each segment [4]. On the other hand, a graphic equalizer is the one in which presets the center frequency and bandwidth of each frequency band, and adjusts the gain according to the instructions [5-8]. The more frequency band is divided, the more accurate the adjustment will be, but the adjustment cost will also increase.

The graphic equalizer can be implemented using the cascade structure [9] or the parallel structure [4] of a set of bandpass filters [9]. In addition, a hybrid of both the cascade structure and the parallel structure of a set of bandpass filters is proposed [10]. However, all the types of the graphic equalizers have a problem that there is an interaction between two adjacent filters [2] [4] [11]. The brute force approach for addressing this issue is via employing a high order FIR filter. However, this results to another problem that the required computational power is very large. Also, the system is suffered from a long delay [6]. For this reason, an IIR filter is used instead.

\footnotetext{
+ Corresponding author. Tel.: +86 203932 2246; fax: +86203932 2252.

E-mail address: yongquanling@gdut.edu.cn
} 
Although the IIR filter is usually suffered from the phase distortion [12], the implementation cost of the IIR filter is much lower than that of the FIR filter under the same specification. Therefore, the IIR filter is preferred in many applications where the phase response is not so important [13] [14]. The traditional method for designing the IIR filter for the audio equalizer is based on the bilinear transformation. However, the frequency response at the high frequency components is distorted [2] [5] [6]. To address this issue, the frequency response at the high frequency components is modified in such a way that it is close to that of the original analog system [15-16]. Nevertheless, this approach cannot guarantee to yield an optimal response.

To address this issue, this paper proposes an optimization approach for designing the equalizer. In particular, the quotient of discrete time Fourier transforms of both the input audio signal and the input signal of the equalizer (It is the output signal of the original system.) is used to represent the frequency response of the transmission system [13]. Then, a special objective function which is based on the error function is minimized under the condition of imposing stability constraint on the optimization problem. Once the optimal solution is obtained, the coefficients of the IIR filter are determined, that is, the equalizer is designed.

The outline of this paper is as follows. The content about optimization problem formulation is discussed in Section 2. The algorithm to solve the optimization problem is presented in Section 3. The computer numerical simulation results are demonstrated in Section 4. Finally, a conclusion is drawn in Section 5.

\section{Optimization Problem Formulation}

\subsection{Transmission System Modeling}

Let the input and output audio signal of the transmission system denoted as $x(n)$ and $y(n)$ for $n=0,1, \ldots L-1$, respectively. And the signals discrete time Fourier transform can be described as follow:

$$
\begin{aligned}
& X(\omega)=\sum_{n=0}^{L-1} x(n) e^{-j n \omega}, \\
& Y(\omega)=\sum_{n=0}^{L-1} y(n) e^{-j n \omega} .
\end{aligned}
$$

Then, the frequency response of the transmission system can be formulated as

$$
H_{s}(\omega)=\frac{Y(\omega)}{X(\omega)}=\frac{\sum_{n=0}^{L-1} y(n) e^{-j n \omega}}{\sum_{n=0}^{L-1} x(n) e^{-j n \omega}} .
$$

If the input signal $x(n)$ passes through the transmission system without any processing, there may be some degree of distortion and some error between the output signal $y(n)$ and the input signal $x(n)$ are produced. Therefore, an equalizer needs to be concatenated after the transmission system for processing to maintain the maximum integrity of the input signal.

\subsection{Constructing Error Function}

The frequency response of an IIR filter based equalizer can be described as

$$
H_{e q}(\omega)=\frac{\sum_{m=0}^{M} b_{m} e^{-j m \omega}}{\sum_{n=0}^{N} a_{n} e^{-j n \omega}},
$$

Here, $M$ and $N$ are the orders of the polynomials of the numerator and the denominator of $H_{e q}(\omega)$, respectively. Also, $b_{m}$ for $m=0,1, \ldots, M$ and $a_{n}$ for $n=0,1, \ldots, N$ are the coefficients in the numerator and the denominator of $H_{e q}(\omega)$, respectively. In order to maintain maximum integrity of the input signal, we have

$$
H_{s}(\omega) \cdot H_{e q}(\omega) \approx 1 \quad \forall \omega \in B_{p},
$$

where $B_{p}$ is the band that needs to be processed. To simplify the problem, only their magnitude response is considered. That is, 


$$
\left|H_{s}(\omega)\right|^{2}\left|\frac{\sum_{m=0}^{M} b_{m} e^{-j m \omega}}{\sum_{n=0}^{N} a_{n} e^{-j n \omega}}\right|^{2} \approx 1 \quad \forall \omega \in B_{p} .
$$

Here, $|\cdot|$ denotes the modulus operator. Define the error function as follow:

$$
E(\omega)=\left|\sum_{n=0}^{N} a_{n} e^{-j n \omega}\right|^{2}-\left|H_{s}(\omega)\right|^{2}\left|\sum_{m=0}^{M} b_{m} e^{-j m \omega}\right|^{2} .
$$

Define

$$
\begin{aligned}
& c_{a}(\omega)=\left[\begin{array}{lll}
1 & \cdots & \cos (N \omega)
\end{array}\right]^{T}, \\
& s_{a}(\omega)=\left[\begin{array}{lll}
0 & \cdots & \sin (N \omega)
\end{array}\right]^{T}, \\
& c_{b}(\omega)=\left[\begin{array}{lll}
1 & \cdots & \cos (M \omega)
\end{array}\right]^{T}, \\
& s_{b}(\omega)=\left[\begin{array}{lll}
0 & \cdots & \sin (M \omega)
\end{array}\right]^{T}, \\
& \vec{a}=\left[\begin{array}{lll}
a_{0} & \cdots & a_{N}
\end{array}\right]^{T}, \\
& \vec{b}=\left[\begin{array}{lll}
b_{0} & \cdots & b_{M}
\end{array}\right]^{T} .
\end{aligned}
$$

Then, (7) can be turned to be

$$
\begin{aligned}
E(\omega)=( & \left.c_{b}^{T}(\omega) \vec{b}\right)^{2}+\left(s_{b}^{T}(\omega) \vec{b}\right)^{2} \\
& -\left(\left(c_{a}^{T}(\omega) \vec{a}\right)^{2}+\left(s_{a}^{T}(\omega) \vec{a}\right)^{2}\right)\left|H_{s}(\omega)\right|^{2}
\end{aligned}
$$

As $E(\omega)$ is defined at each frequency, the total error function is minimized instead. That is,

$$
\begin{aligned}
& J(\vec{b}, \vec{a})=\int_{\omega \in B_{P}}|E(\omega)| d \omega \\
& =\int_{\omega \in B_{P}} \mid\left(c_{b}^{T}(\omega) \vec{b}\right)^{2}+\left(s_{b}^{T}(\omega) \vec{b}\right)^{2} \\
& -\left(\left(c_{a}^{T}(\omega) \vec{a}\right)^{2}+\left(s_{a}^{T}(\omega) \vec{a}\right)^{2}\right)\left|H_{s}(\omega)\right|^{2} \mid d \omega
\end{aligned}
$$

is minimized. In order to prevent a frequency having a large overshoot, the following condition is imposed to the design problem. That is,

$$
|E(\omega)|=\left|\begin{array}{l}
\left(c_{b}^{T}(\omega) \vec{b}\right)^{2}+\left(s_{b}^{T}(\omega) \vec{b}\right)^{2} \\
-\left(\left(c_{a}^{T}(\omega) \vec{a}\right)^{2}+\left(s_{a}^{T}(\omega) \vec{a}\right)^{2}\right)\left|H_{s}(\omega)\right|^{2}
\end{array}\right| \leq \delta(\omega) \forall \omega \in B_{p}
$$

Here, $\delta(\omega)>0$ for $\forall \omega \in B_{p}$ is the upper bound on acceptable error. This is equivalent to following conditions:

$$
\left(\begin{array}{l}
\left(c_{b}^{T}(\omega) \vec{b}\right)^{2}+\left(s_{b}^{T}(\omega) \vec{b}\right)^{2} \\
-\left(\left(c_{a}^{T}(\omega) \vec{a}\right)^{2}+\left(s_{a}^{T}(\omega) \vec{a}\right)^{2}\right)\left|H_{s}(\omega)\right|^{2}
\end{array}\right)-\delta(\omega) \leq 0 \forall \omega \in B_{p}
$$

and

$$
\left(\begin{array}{l}
-\left(c_{b}^{T}(\omega) \vec{b}\right)^{2}-\left(s_{b}^{T}(\omega) \vec{b}\right)^{2} \\
+\left(\left(c_{a}^{T}(\omega) \vec{a}\right)^{2}+\left(s_{a}^{T}(\omega) \vec{a}\right)^{2}\right)\left|H_{s}(\omega)\right|^{2}
\end{array}\right)-\delta(\omega) \leq 0 \forall \omega \in B_{p} .
$$

Unfortunately, the results obtained by these constraints do not guarantee the stability of the system. To tackle the stability issue, the stability constraint [3] as follow is imposed in the optimization problem: 


$$
\operatorname{Re}\left(\sum_{n=0}^{N} a_{n} e^{-j n \omega}\right)<0 \quad \forall \omega \in B_{p}
$$

Then, the optimization problem can be described as:

$$
\begin{array}{ll} 
& \left.\min _{\vec{a}, \vec{b}} \int_{\omega \in B_{P}} \mid \begin{array}{l}
\left(c_{b}^{T}(\omega) \vec{b}\right)^{2}+\left(s_{b}^{T}(\omega) \vec{b}\right)^{2} \\
-\left(\left(c_{a}^{T}(\omega) \vec{a}\right)^{2}+\left(s_{a}^{T}(\omega) \vec{a}\right)^{2}\right)\left|H_{s}(\omega)\right|^{2} \mid d \omega
\end{array}\right)-\delta(\omega) \leq 0 \forall \omega \in B_{p}, \\
\text { s.t. }\left(\begin{array}{l}
\left(c_{b}^{T}(\omega) \vec{b}\right)^{2}+\left(s_{b}^{T}(\omega) \vec{b}\right)^{2} \\
-\left(\left(c_{a}^{T}(\omega) \vec{a}\right)^{2}+\left(s_{a}^{T}(\omega) \vec{a}\right)^{2}\right)\left|H_{s}(\omega)\right|^{2}
\end{array}\right)-\delta(\omega) \leq 0 \forall \omega \in B_{p}, \\
\left(\begin{array}{l}
-\left(c_{b}^{T}(\omega) \vec{b}\right)^{2}-\left(s_{b}^{T}(\omega) \vec{b}\right)^{2} \\
+\left(\left(c_{a}^{T}(\omega) \vec{a}\right)^{2}+\left(s_{a}^{T}(\omega) \vec{a}\right)^{2}\right)\left|H_{s}(\omega)\right|^{2}
\end{array}\right) \\
\operatorname{Re}\left(\sum_{n=0}^{N} a_{n} e^{-j n \omega}\right)<0 \quad \forall \omega \in B_{p} .
\end{array}
$$

\section{Solving Optimization Problem}

It is worth noting that the genetic algorithm is a numerical optimization method inspired by the biological evolution of the genes via a natural selection mechanism. The algorithm consists of several procedures. They are the population initialization, the objective functional evaluation, the selecting operation, the crossover operation, the mutation operation and testing the stopping criterion.

\section{Step 1: Population initialization}

It is worth noting that the global optimal solution may not be found if the population size is too small. On the other hand, the required computational power is large if the population size is large. In this paper, the population size is set at 100 . Here, each individual in the population represents a vector that has the same dimension of $\left[\begin{array}{ll}\vec{a}^{T} & \vec{b}^{T}\end{array}\right]^{T}$ and satisfies all the constraints.

\section{Step 2: Objective functional evaluation}

Calculate the objective functional value of each individual in the population set.

Step 3: Selection operation

Eliminate the individuals where the objective functional values are too large. In this paper, the retention rate is 0.1 . That means, only 10 individuals with small objective functional values are retained.

\section{Step 4: Crossover operation}

The remaining individuals will be paired up randomly. The same crossover point is set randomly to the individuals in the same pair. Then, divide each vector into two parts according to the crossover point. Next, exchange the parts of the vectors to produce two new individuals. These operations are repeated until the total number of the generated individuals is equal to the total number of the individuals eliminated in Step 3.

\section{Step 5: Mutation operation}

Each individual generated in step 4 has elements reset randomly between 0 and 1 according to the mutational probability. It is worth noting that the optimal solution cannot be found if the mutational probability is too high. On the other hand, it cannot kick out from the local optimal solution if the mutational probability is too low. In this paper, the mutational probability is set at 0.02 .

\section{Step 6: Testing the stopping criterion}

Define the stopping criterion as a condition that the absolute change of the objective functional values between two consecutive iterations is smaller than a predefined threshold. Repeat Step 2 to Step 6 until the stopping criterion is reached. 


\section{Computer Numerical Simulation Results}

Without loss of the generality, assume that the input signal of the original system without connecting the equalizer is an ideal white Gaussian noise, that is, $x(n)$ is an ideal white Gaussian noise. And the signal $y(n)$ is the output signal of transmission system. Then the frequency response of the transmission system can be simply represented as quotient of the discrete time Fourier transform of $x(n)$ and $y(n)$. By constructing a specific objective function with overshooting constraints and stability constraint discussed in Section II and applying genetic algorithm to solve the optimization problem, an optimal result $\vec{a}_{o p}, \vec{b}_{o p}$ can be obtained.
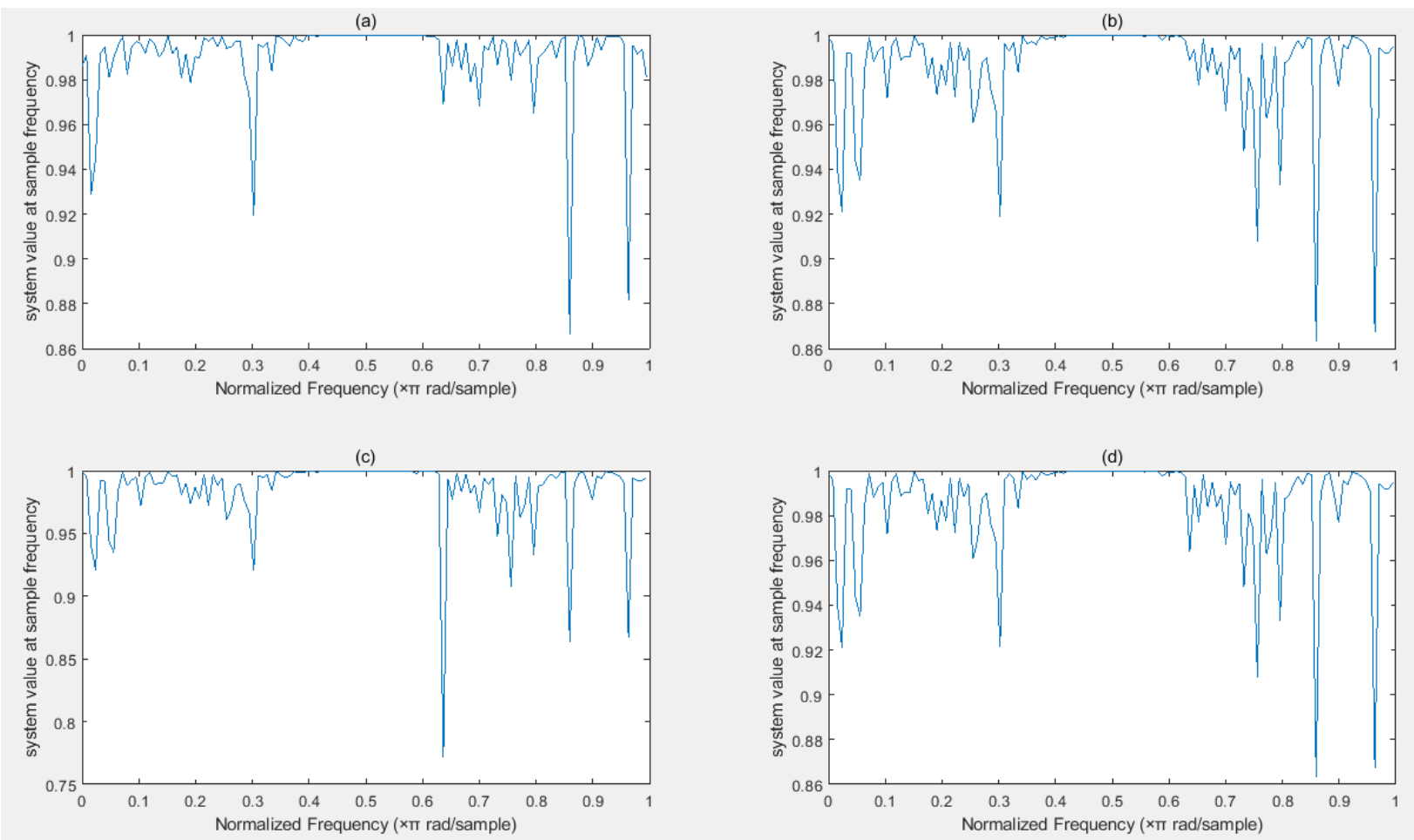

Fig. 1: The product of the magnitude responses of the joint original system and the equalizer based on (a) our designed IIR filter, (b) the Butterworth filter, (c) the Chebyshev filter and (d) the elliptic filter.

To demonstrate the effectiveness of our deigned equalizer, the existing equalizers based on the Butterworth filter, the Chebyshev filter and the elliptic filter with the same order which is equal to 73 in this paper, are employed for the comparisons. Figure 1 shows the products of the magnitude responses of the joint original system and the equalizers based on our designed IIR filter, the Butterworth filter, the Chebyshev filter and the elliptic filter. It can be seen from Figure 1 that the product of the magnitude responses of the joint original system and the equalizers based on our designed IIR filter is between 0.98 and 1 for nearly the whole frequency band, while those designed by the Butterworth filter, the Chebyshev filter and the elliptic filter vary severely in the frequency band between $0.7 \pi$ and $0.8 \pi$.According to (25), as the closer the curve to the constant 1 for the whole frequency band refers to the better equalizer performance, our designed IIR filter outperforms these existing filters. Moreover, the Butterworth filter, the Chebyshev filter and the elliptic filter are unstable. On the other hand, our designed IIR filter is guaranteed to be stable because the stability constraint is imposed in our design.

\section{Conclusion}

This paper proposes a functional inequality constrained optimization approach for designing the equalizer. It is found that the product of the magnitude responses of the joint original system and the equalizer based on our designed IIR filter is between 0.98 and 1 for nearly the whole frequency band. Also, our designed IIR filter is guaranteed to be stable. 


\section{Acknowledgements}

This paper was supported partly by the National Nature Science Foundation of China (no. U1701266, no. 61372173 and no. 61671163), the Team Project of the Education Ministry of the Guangdong Province (no. 2017KCXTD011), the Guangdong Higher Education Engineering Technology Research Center for Big Data on Manufacturing Knowledge Patent (no. 501130144), the Guangdong Province Intellectual Property Key Laboratory Project (no. 2018B030322016) and Hong Kong Innovation and Technology Commission, Enterprise Support Scheme (no. S/E/070/17).

\section{References}

[1] D.A. Bohn, "Operator adjustable equalizers: an overview," The 6th Audio Engineering Society International Conference on Sound Reinforcement, pp. 369-381, 1988.

[2] V. Välimäki and J. Reiss, "All about audio equalization: solutions and frontiers," Applied Sciences, vol. 6, no. 5, pp. 129, 2016.

[3] M. Virgulti, S. Cecchiand F. Piazza, "IIR filter approximation of an innovative digital audio equalizer," The International Symposium on Image and Signal Processing and Analysis, ISPA, pp. 410-415, 2013.

[4] J.Ramo, V. Valimaki and B. Bank, "High-precision parallel graphic equalizer," IEEE/ACM Transactions on Audio, Speech and Language Processing, vol. 22, no. 12, pp. 1894-1904, 2014.

[5] T. van Waterschoot and M. Moonen, "A pole-zero placement technique for designing second-order IIR parametric equalizer filters," IEEE Transactions on Audio, Speech and Language Processing, vol. 15, no. 8, pp. 2561-2565, 2007.

[6] S.Sarkka and A. Huovilainen, "Accurate discretization of analog audio filters with application to parametric equalizer design," IEEE Transactions on Audio, Speech and Language Processing, vol.19, no. 8, pp. 2486-2493, 2011.

[7] J.D.Reiss, "Design of audio parametric equalizer filters directly in the digital domain," IEEE Transactions on Audio, Speech and Language Processing, vol. 19, no. 6, pp. 1843-1848, 2011.

[8] P. Regalia and S. Mitra, "Tunable digital frequency response equalization filters," IEEE Transactions on Acoustics, Speech and Signal Processing, vol. ASSP-35, no. 1, pp. 118-120, 1987.

[9] J.Ramo and V. Valimaki, “Optimizing a high-order graphic equalizer for audio processing," IEEE Signal Processing Letters, vol. 21, no. 3, pp. 301-305, 2014.

[10] M. Erne and C. Heidelberger, "Design of a DSP-based 27 band digital equalizer," The $90^{\text {th }}$ Convention of the Audio Engineering Society, 1991.

[11] J.A.Belloch and V. Valimaki, "Efficient target-response interpolation for a graphic equalizer," The IEEE International Conference Acoustics, Speech and Signal Processing, ICASSP, pp. 564-568, 2016.

[12] C.Y.F. Ho, B.W.K. Ling, Z.W. Chi, M.Shik-Bahaei, Y.Q. Liu and K.L.Teo, "Design of near allpass strictly stable minimal phase real valued rational IIR filters," IEEE Transactions on Circuits and Systems-II: Express Briefs, vol. 55, no. 8, pp. 781-785, 2008.

[13] C.Y.F. Ho, B.W.K. Ling, Y.Q. Liu, P.K.S. Tam and K.L.Teo, "Optimal design of magnitude responses of rational infinite impulse response filters," IEEE Transactions on Signal Processing, vol. 54, no. 10, pp. 4039-4046, 2006.

[14] L.S.H.Ngia, "Recursive identification of acoustic echo systems using orthonormal basis functions," IEEE Transactions on Speech and Audio Processing, vol. 11, no. 3,pp. 278-293,2003.

[15] S.J. Orfanidis, "Digital parametric equalizer design with prescribed Nyquist-frequency gain," Journal of Audio Engineering Society, vol. 45, no. 6, pp. 444-455, 1997.

[16] P.Lin, W.Kuang, Y. Liu and B.W.K. Ling, "Grouping and selecting singular spectrum analysis components for denoisingvia empirical mode decomposition approach," Circuits, Systems and Signal Processing, vol. 38, no. 1, pp. 356-370, 2019. 